\title{
Structural response of submerged air-backed plates by experimental and numerical analyses
}

\author{
Lloyd Hammond $^{\mathrm{a}}$ and Raphael Grzebieta ${ }^{\mathrm{b}}$ \\ ${ }^{\mathrm{a}}$ Aeronautical and Maritime Research Laboratory, \\ Defence Science and Technology Organisation, \\ Melbourne Victoria, 3001, Australia \\ Tel.: +6139626 8754; Fax: +6139626 8999; \\ E-mail: lloyd.hammond@dsto.defence.gov.au \\ ${ }^{\mathrm{b}}$ Department of Civil Engineering, Monash \\ University, Clayton, Victoria, Australia \\ Tel.: +61399054970
}

Received 9 December 1998

Revised 17 October 1999

This paper presents the results of a series of small-scale underwater shock experiments that measured the structural responses of submerged, fully clamped, air-backed, steel plates to a range of high explosive charge sizes. The experimental results were subsequently used to validate a series of simulations using the coupled LS-DYNA/USA finite element/boundary element codes. The modelling exercise was complicated by a significant amount of local cavitation occurring in the fluid adjacent to the plate and difficulties in modelling the boundary conditions of the test plates. The finite element model results satisfactorily predicted the displacementtime history of the plate over a range of shock loadings although a less satisfactory correlation was achieved for the peak velocities. It is expected that the predictive capability of the finite element model will be significantly improved once hydrostatic initialisation can be fully utilised with the LS-DYNA/USA software.

Keywords: Underwater shock, underwater experiments, LSDYNA/USA, finite element, structural response, dynamic loading, air-backed plates

\section{Introduction}

The response of maritime vessel structures in the vicinity of an underwater explosion is of concern, and must be established for first-of-class vessels. This con- cern arises primarily in relation to the dynamic structural response and the subsequent transmitted response to shock hardened equipment and systems within the vessel. A modelling and simulation capability for predicting structural and equipment response to an underwater explosion is highly desirable and of value to naval architects and to the designers of onboard equipment.

The structural response of small-scale structures to underwater explosions is being investigated by the Defence Science and Technology Organisation with the ultimate purpose of developing a numerical capability for predicting the response of maritime vessels subjected to a far-field underwater explosion attack, such as that caused by a sea-mine or depth charge. The structural response of a fully submerged air-backed plate to a far-field underwater explosion involves several contributing phenomena, including the direct shock wave, sea-surface and sea-bed reflected shock waves, local hull and bulk cavitation effects, and gas-bubble pulsation and migration effects. Fluid-structure coupling effects caused by the deforming plate interacting with the incident pressure load also complicate the structural response, since the plate surface scatters a significant portion of the incident pressure wave back into the water, which in turn, interacts with the remainder of the incoming pressure wave. This complex coupling problem can cause local cavitation of the water at close proximity to the plate surface, which will eventually collapse and possibly reload the plate.

The work presented in this paper reports the experimental and numerical analyses undertaken as part of the investigation into the fluid-structure interaction of the direct shock wave with a fully submerged, air-backed, square plate fully clamped to a rigid box structure. The numerical simulations were performed using the LSDYNA non-linear, dynamic analysis code [3] with the USA code [1,2]. Data from these simulations were used for predicting local and global structural responses for a range of scenarios of varying charge sizes and stand- 
off distances. Structural responses for these scenarios were measured in a series of far-field underwater shock tests. The tests were designed so that only the effects of the direct shock wave and local cavitation had to be considered.

\section{Experiment design}

The underwater explosion tests were designed to provide detailed information with respect to strains, pressures, accelerations and displacements for air-backed, square, steel plates loaded by the underwater shock waves that could be compared with the results of those generated by finite element analyses. In addition, tensile tests were carried out on test coupons cut from the same D36 steel plate used in the underwater explosion tests. The results of the coupon tests were used for specifying material properties in the finite element modelling.

The underwater shock tests were performed on a number of unstiffened, $3 \mathrm{~mm}$ thick, square, D36 steel plates with edge lengths of $780 \mathrm{~mm}$, clamped to a reinforced steel box, henceforth referred to as a shock rig. The shock rig was suspended midway beneath a pair of floating pontoons at the Aeronautical and Maritime Research Laboratory's Epping Quarry testing facility (approximately $20 \mathrm{~m}$ deep and $100 \mathrm{~m}$ diameter).

Twenty tests were conducted, providing sufficient data for determining trends for various structural parameters. The charge size and standoff distances were selected to cover a range of elastic and elastic-plastic structural responses. A nominal standoff distance of $4 \mathrm{~m}$ was selected. This was so that the standoff distance would be significantly greater than the expected gas-bubble diameter, thus avoiding bubble collapse on to the structure, which in turn minimised gas-bubble related structural response. The centrally detonated, cylindrical pentolite explosives were arranged perpendicular to and at the same depth as the centre of the test plates, i.e. $2.3 \mathrm{~m}$.

The shock rig was suspended below two floating pontoons spaced $5 \mathrm{~m}$ apart as shown in Fig. 1. The pontoons, which were connected by two, parallel, steel I-beams bolted to the top surface of each pontoon.

The flat plates were bolted to the top, open face of the shock rig, between two flange plates as illustrated in Fig. 2. A $3 \mathrm{~mm}$ natural rubber gasket was positioned between the test plate and the flange of the shock rig to ensure water-tightness within the rig. This securing arrangement was designed to provide a fully clamped (i.e. built-in edge) boundary condition for the test plates.

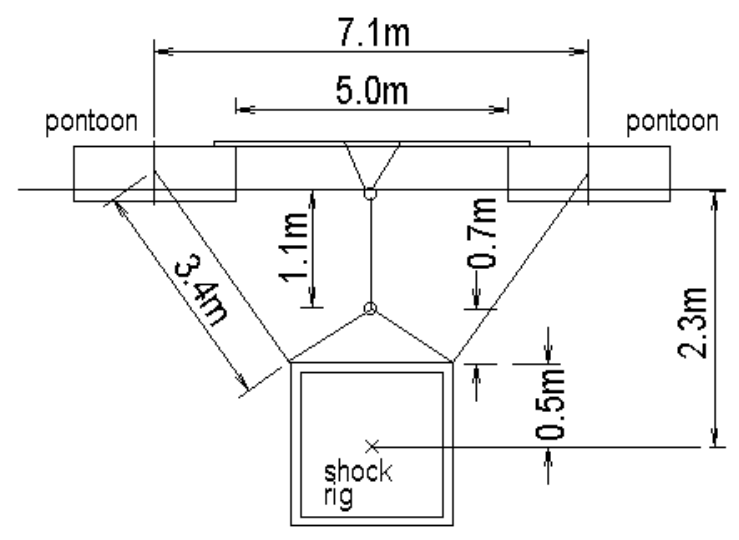

Fig. 1. Schematic drawing showing the chain arrangement used to secure the shock rig with respect to the pontoons. The charge is located at $2.3 \mathrm{~m}$ depth, perpendicular to the test plate centre.

\section{Experiment}

Seventeen channels of data were acquired consisting of acceleration, strain, pressure and displacement. The selection of transducers included 12 panel mounted transducers, including five flush-mounted airblast gauges (P1 to P5), two accelerometers (A1 and A2), four $8 \mathrm{~mm}$ single element $350 \Omega$ precision strain gauges (SI1, SI2, SX1, SX2) and one displacement transducer (D1) as shown in Fig. 3. A third accelerometer, A3, was positioned on the rear of the shock rig and four free-field pressure gauges were suspended in the water at strategic locations around the shock rig.

Two displacement transducers were used for measurement of the deflection of the test plate centre. An optical displacement transducer (ODT) was used initially, but as the ODT's measurement range is limited to $50 \mathrm{~mm}$ (i.e. $\pm 25 \mathrm{~mm}$ ), larger deflections necessitated the use of an alternative transducer. In this case, a mechanical potentiometer displacement transducer (designed and built at the Underwater Explosions Research Department, Naval Surface Weapons Center, Carderock, USA) with a measuring range of $180 \mathrm{~mm}$ (i.e. $\pm 90 \mathrm{~mm}$ ), was utilised.

Free-field pressure measurements for the twenty tests were used to calculate similitude parameters for the pentolite explosives detonated at the Aeronautical and Maritime Research Laboratory quarry site. The resultant similitude parameters for peak pressure, time constant, impulse and energy are: 


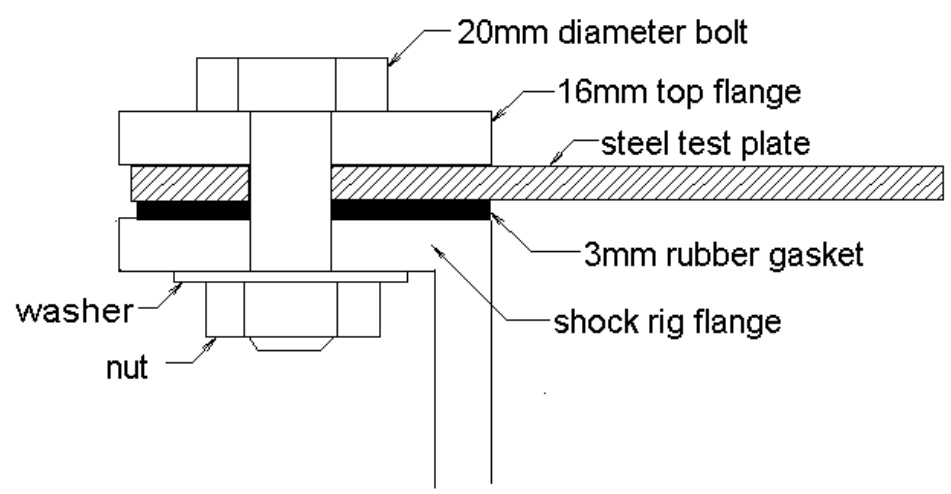

Fig. 2. Schematic drawing showing the arrangement employed for securing the test plate to the shock rig.

$$
\begin{array}{ll}
\text { Pressure: } & \mathrm{P}=45.6\left(\mathrm{~W}^{1 / 3} / \mathrm{R}\right)^{1.11} \mathrm{MPa} \\
\text { Time Constant: } & \theta / \mathrm{W}^{1 / 3}= \\
& 0.097\left(\mathrm{~W}^{1 / 3} / \mathrm{R}\right)^{-0.15} \mathrm{~ms} \mathrm{~kg}^{1 / 3} \\
\text { Impulse: } & \mathrm{I} / \mathrm{W}^{1 / 3}= \\
& 5148\left(\mathrm{~W}^{1 / 3} / \mathrm{R}\right)^{0.93} \mathrm{~Pa}-\mathrm{s} \mathrm{kg}^{1 / 3} \\
\text { Energy: } & \mathrm{E} / \mathrm{W}^{1 / 3}= \\
& 70922\left(\mathrm{~W}^{1 / 3} / \mathrm{R}\right)^{2.05} \mathrm{~m}-\mathrm{Pa} / \mathrm{kg}^{1 / 3}
\end{array}
$$

where $W$ and $R$ are charge weight and standoff distance, respectively. These similitude equations were used for determining the loading parameters for the finite element models.

\section{Finite element model}

A quarter-symmetry finite element model of the shock rig described in the experiment in the previous section was constructed using MSC/Patran (v7.5) preprocessing software. The finite element model of the shock rig consisted of 2500 Hughes-Lui formulation shell elements. The D36 ship steel for the test plates and the mild steel of the shock rig was modelled using the LS-DYNA Material 3, which has a bi-linear stressstrain model for modelling the elastic/plastic nature of a material. The D36 plate properties had previously been determined by a series of tensile coupon tests. The Material-3 parameters used in the finite element model were:

$$
\begin{array}{ll}
\text { Density: } & \rho=7850 \mathrm{~kg} / \mathrm{m}^{3} \\
\text { Young's Modulus: } & E=211,639 \mathrm{~kg} / \mathrm{m}^{2} \\
\text { Poisson's Ratio: } & \nu=0.3 \\
\text { Yield Stress (static): } & \sigma_{y}=411 \mathrm{MPa} \\
\text { Tangent Modulus: } & E_{t}=500 \mathrm{MPa}
\end{array}
$$

Some simplifications were made in modelling the shock rig structure, particularly in relation to the clamping arrangement of the plate (refer Fig. 4). The flange section of the shock rig, which included the upper and lower flanges, the $3 \mathrm{~mm}$ test plate and the $3 \mathrm{~mm}$ rubber gasket that extended beyond the side faces of the shock rig, were smeared into one layer of shell elements. The fluid surrounding the box was modelled using an elastic fluid material (Material-1) and required 29232 fluid elements. Material-1 parameters used in the finite element model were:

$$
\begin{array}{ll}
\text { Density: } & \rho=1000 \mathrm{~kg} / \mathrm{m}^{3} \\
\text { Bulk Modulus: } & G=2167 \mathrm{~kg} / \mathrm{m}^{2} \\
\text { Cavitation parameter: } & C P=0.10 \mathrm{MPa}
\end{array}
$$

The fluid elements are $10 \mathrm{~mm}$ in length in the direction of the acoustic wave propagation. This element length was chosen after power spectrum analysis of the shortest duration pressure load (i.e. $\theta=0.033 \mathrm{~ms}$ ). The criterion for selection of the element size is that it must be at least one tenth of the minimum wavelength, $\lambda$, of each frequency component in order to faithfully simulate the propagation of the acoustic pressure wave. Additionally, $85 \%$ of the "power" of the pressure wave must remain intact after propagation through the mesh. A Fourier power spectrum was generated such that the principal frequency components could be determined for this purpose. The $85 \%$ figure is nominal and based upon balancing modelling requirements against computation time. Retaining power beyond $85 \%$ requires relatively larger mesh densities that became unrealistic for modelling purposes. Assuming an $85 \%$ power capture, the maximum element dimension must be less than $0.15 \times c \theta$, where $c$ and $\theta$ are the acoustic speed and pressure decay constants, respectively. Convergence studies were also performed on simple 1D fluid models to verify the selection of element size. Exponential pressure waves were propagated along a 1D column of fluid elements of various mesh densities to determine the relative integrity loss of each acoustic wave. These studies confirmed the choice of element size and 
showed that further reductions in the element size did not significantly alter the pressure wave profile.

The Arbitrary Lagrangian Eulerian (ALE) capability was applied to the entire fluid region in the finite element model after each LS-DYNA time step. This was done so as to avoid distortion or elongation of the fluid elements arising from the deflections of the test plate elements at the Eulerian-Lagrangian (fluid-structure) interface of the finite element model.

\section{Hydrostatic initialisation}

A preliminary LS-DYNA run was performed to hydrostatically initialise the finite element model. This was achieved by initialising the stresses of all fluid and structural elements and additionally, by applying a hydrostatic pressure load to all outer fluid element segments for the duration of the run. For this model, it was found that $150 \mathrm{~ms}$ provided sufficient time for the fluid pressures to converge to a uniform value over the fluid region. Similarly, the structural motions, particularly those of the relatively compliant test plate, were allowed to settle to a quasi-static value. The output from this run was intended to provide initial stresses and revised nodal coordinate data for use in the input deck for subsequent LS-DYNA/USA (version 940.2) simulations described in the following section. However, the LS-DYNA/USA software used at this time had errors, which prevented the use of this data (Note: this error only occurred for the LS-DYNA/USA coupled codes and was subsequently corrected in later versions). As a consequence of this problem, an alternative method for hydrostatic initialisation was used, as described in the following section.

\section{LS-DYNA/USA simulations}

The quarter symmetry model shown in Fig. 4(a) has $4480 \mathrm{DAA}_{1}$ (first order doubly asymptotic approximation) elements coincident with all exterior fluid segments (excluding symmetry boundaries). Eight finite element simulations were performed, each with different charge-weight and standoff distance parameters. The standoff distance, peak pressure and decay constants for each simulation were selected to match the equivalent experimental test, shown in Table 1. $50 \mathrm{~ms}$ of structural response were generated for each of the eight simulations. Some simulations were run for an extended period of $200 \mathrm{~ms}$ so as to determine the fre-

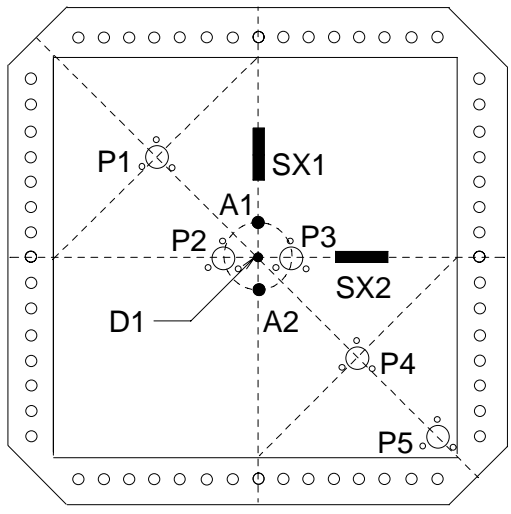

Fig. 3. Schematic diagram showing positioning of transducers on the test plates. P1 to P5 indicate the locations of the flush-mounted pressure transducers; A1 and A2, the accelerometers; SX1 and SX2, the surface-mounted strain gauges (aligned in the direction shown) and D1, the displacement transducer. Two additional strain gauges, SI1 and SI2 are mounted on the reverse, dry surface of the test plates in back-to-back positions to their SX1 and SX2 strain gauge counterparts, respectively.

Table 1

Experimental parameters used for the eight LS-DYNA/USA simulations

\begin{tabular}{ccccc}
\hline $\begin{array}{c}\text { Simulation } \\
\text { Number }\end{array}$ & $\begin{array}{c}\text { Charge } \\
\text { Weight } \\
(\mathrm{g})\end{array}$ & $\begin{array}{c}\text { Standoff } \\
\text { Distance } \\
(\mathrm{m})\end{array}$ & $\begin{array}{c}\text { Peak } \\
\text { Pressure } \\
(\mathrm{MPa})\end{array}$ & $\begin{array}{c}\text { Decay } \\
\text { Constant } \\
(\mathrm{ms})\end{array}$ \\
\hline 1 & 11.2 & 3.7 & 2.025 & 0.033 \\
2 & 36.3 & 3.66 & 3.167 & 0.046 \\
3 & 84.7 & 3.63 & 4.373 & 0.058 \\
4 & 164 & 3.63 & 5.584 & 0.071 \\
5 & 283 & 3.61 & 6.875 & 0.082 \\
6 & 463 & 3.6 & 8.274 & 0.095 \\
7 & 668 & 3.48 & 9.84 & 0.104 \\
8 & 1000 & 3.46 & 11.5 & 0.117 \\
\hline
\end{tabular}

quency response of the target plate. The incident pressure load was initiated at the DAA boundary and propagated through the fluid mesh to the plate and surrounding shock rig structure.

Results from the eight LS-DYNA/USA simulations were analysed using the LS-DYNA postprocessor, LSTAURUS. In each case, the incident pressure wave interacted with the $3 \mathrm{~mm}$ steel plate in such a way as to promote local cavitation in the fluid region directly adjacent to the plate (e.g. see Fig. 5). The time of cavitation initiation $\left(t_{c a v}\right)$ and the duration of cavitation $\left(\tau_{\text {cav }}\right)$ are important parameters in the loading and subsequent structural response of the plate.

The introduction of the local cavitation zone adjacent to the plate, caused by the interaction of the incident and scattered waves, effectively reduces the load time and subsequently the overall load on the plate. The existence and the extent of the local cavitation zone 


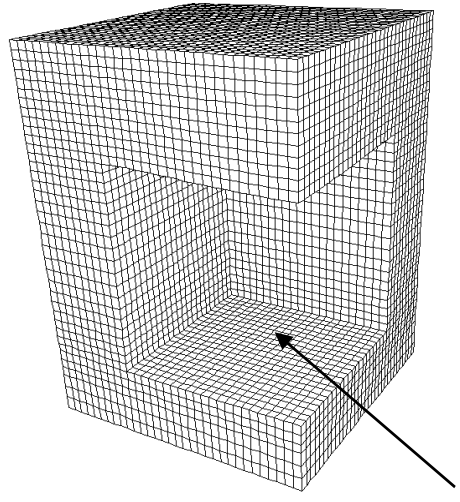

(a)

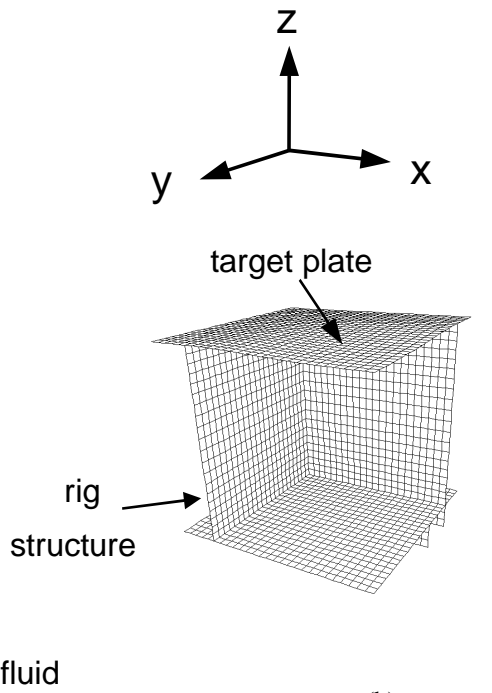

(b)

Fig. 4. Hydrostatically initialised, quarter-symmetry, finite element meshes for the (a) fluid volume surrounding the shock rig and (b) the shock rig itself.



Fig. 5. An LS-TAURUS plot showing the propagation of the incident pressure wave (in the negative z-direction), through the fluid mesh approximately $90 \mu$ s after the pressure wave (dark shade) has impinged on the plate surface (simulation \#4). The simulation shows the local cavitation zone (white shade) extending over the entire region of the test plate and approximately $25 \mathrm{~cm}$ in front of the plate. The rig structure mesh has been excluded from this diagram.

is dependent upon the ambient hydrostatic pressure, the magnitude and duration of the load, and the plate velocity after the pressure load has interacted with the plate. As a consequence, the accuracy of the finite element model is largely dependent upon the initial pressure condition of the fluid in the model, prior to application of the pressure load at the DAA boundary. This is the rationale for hydrostatically initialising the model prior to applying the shock load.

Hydrostatic initialisation was resolved by using small explosive charges in front and behind the shock rig to simulate ambient hydrostatic pressure. The peak pressure for these two quasi-hydrostatic field charges was set at the ambient hydrostatic pressure of $0.022 \mathrm{MPa}$ and their decay time was set at $10^{4} \mathrm{~ms}$, thus resembling a hydrostatic pressure.

It was desirable to allow the model parameters to converge to a steady value before initiating the principal shock load. However, due to limitations in the USA code, it was not possible to delay the principal pressure load by more than $7 \mathrm{~ms}$ (without losing pressure profile information). Consequently, the pressure field in the surrounding fluid had not fully equalised before the propagation of the principal load was initiated. As a result, local cavitation in the model was not accurately simulated. The disparity in cavitation closure times, which may be partly or wholly due to the model not being hydrostatically initialised, are shown in a comparison of the experimental and finite element fluid pressures adjacent to the centre of the test plate in Fig. 6.

The nature of the local cavitation not only affects the incident pressure load, but also affects the manner in which the plate responds after cavitation is initiated. In all eight simulations, the test plate deflected inwards into the air cavity of the shock rig and then returns back, through the plate's initial rest position, into the water region. If the water has cavitated at the time the plate moves outwards from the box, then the surrounding fluid had minimal effect on the plate response until the cavitation zone closes. In the eight simulations performed, all structural response parameters tend to 


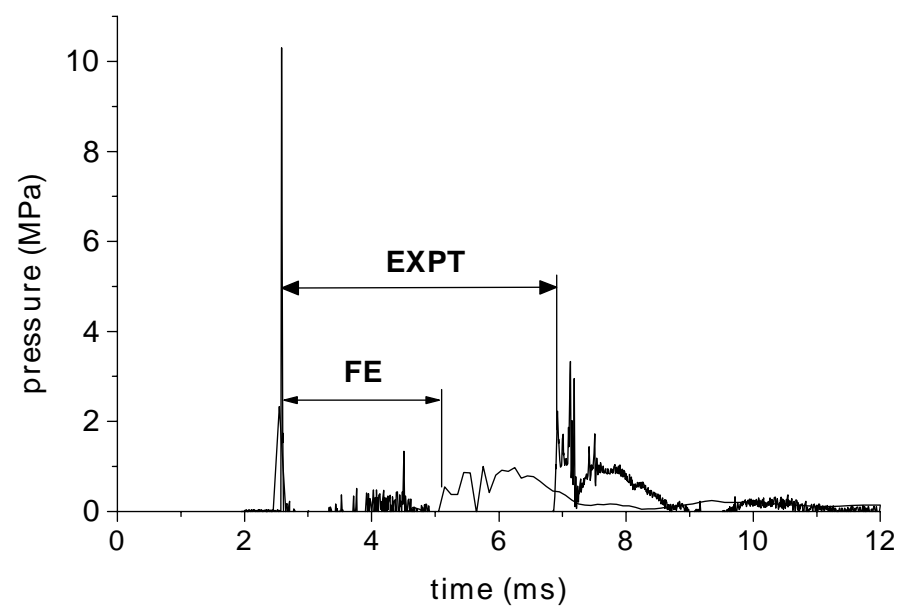

Fig. 6. Comparison of cavitation duration for the finite element model (simulation \#4) and the related experimental data from event 8 (EXPT) adjacent to the test plate centre (pressure gauge: P2). The cavitation closure for the finite element model occurs almost 2 ms earlier than for the experiment.



Fig. 7. Comparison of experimental (EXPT) and finite element results for the displacement of the test plate centre. (The experimental data was acquired using an optical displacement transducer).

be underestimated by the finite element model. For example, the centre of the plate deflects inwards approximately $18 \mathrm{~mm}$ for the finite element model shown in Fig. 7, compared to $23 \mathrm{~mm}$ for the equivalent experimental measurement. The time that the plate spends extended outwards into the fluid region is less for the finite element model than for the equivalent experimental data. This is expected since the finite element model underestimates the duration of cavitation as explained above.

The applicability of the finite element model can also be judged from correlation in frequency response of the test plate between experimental and finite element data. For example, for the extended $200 \mathrm{~ms}$ finite element simulation \#4, the average natural frequency response of the air-backed plate in the fluid (for the period after the initial structural response) is approximately $37.5 \mathrm{~Hz}$, compared to $36 \mathrm{~Hz}$ for the experimental data. The minor discrepancy in this value is likely to be due to discrepancies in the boundary conditions for the edge of the test plate between the finite element model and the experimental shock rig.

Double-integration and bandpass filtering of the accelerometer data were used to obtain peak displacement values for the test plate centre as shown in Fig. 8(a). Displacement data was also available from a limited 


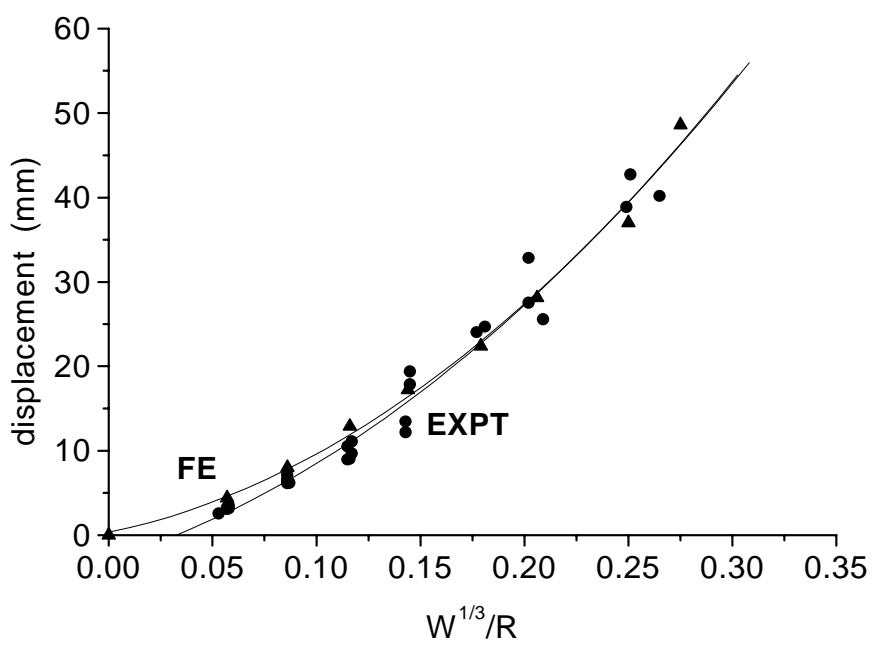

(a)

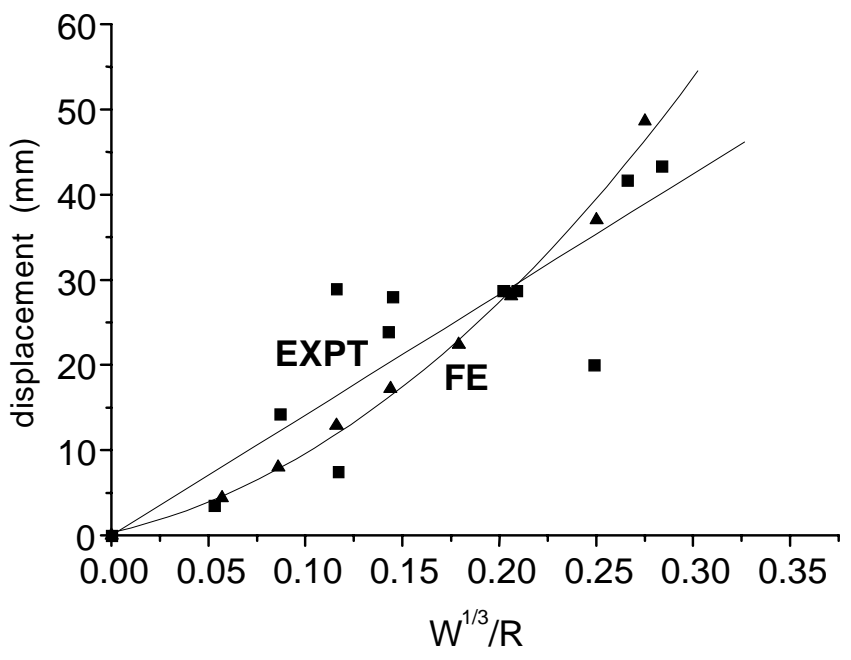

(b)

Fig. 8. Comparison of finite element results for deflection of the test plate centre versus experiment data (EXPT) for (a) the doubly integrated accelerometer data and (b) the displacement transducer data.

number of events from two displacement transducers. The displacement transducers proved less reliable than other transducers used and in particular, for the case of the ODT, data was only available for a limited range of displacements. However, this was the first occasion that the ODT prototype was tested in an underwater shock environment and many problems with the device were resolved during the course of these tests. A comparison of the displacement data for the eight finite element simulations is compared with the displacement transducer data in Fig. 8(b). Least-squares regression methods have been applied to the finite element and experimental data. The accelerometer data and the finite element simulation data was best approximated using a second order polynomial fit. There was insufficient data for the displacement transducer to apply a second order polynomial fit, so a linear least-squares fit was applied.

Integration and bandpass filtering of the accelerometer data were also used to generate velocity-time profiles. From these profiles, peak velocities of the plate centre were obtained and compared with finite element simulation data, as shown in Fig. 9. The finite element model velocities noticeably under predict the velocities calculated from the accelerometer data as charge size increases. There is no immediate justification for this 


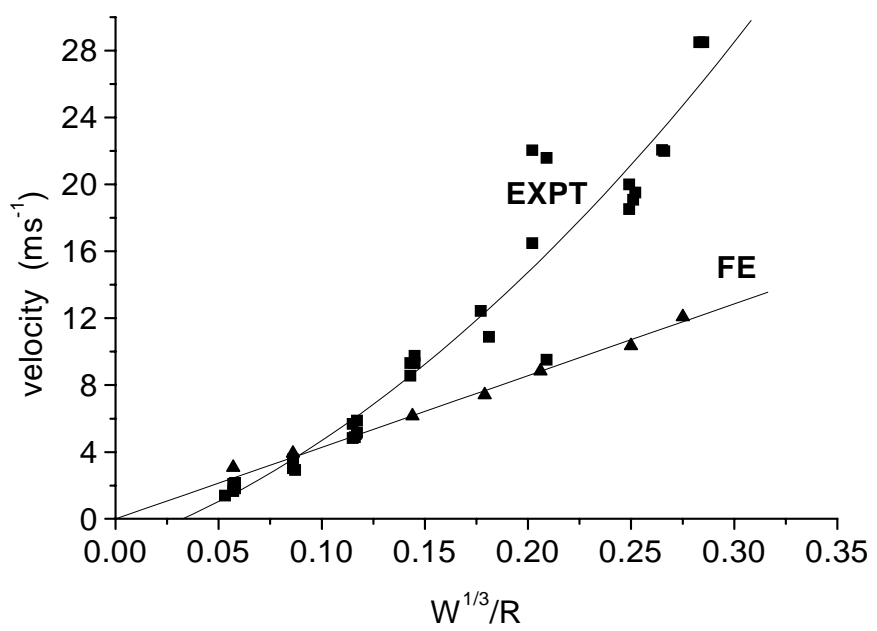

Fig. 9. Comparison of finite element and experiment (EXPT) results for velocity of the test plate centre.

discrepancy, given the excellent agreement between experimental and finite element displacement values. However, differences in the boundary conditions of the test plate between the finite element model and the experiment are likely to generate discrepancies in the resultant velocity data. Strain rate effects, which were determined from the experimental data, were considered to be insignificant since their values were found to be reasonably low (i.e. typically less than $0.5 \mathrm{~s}^{-1}$ ).

\section{Discussion}

A limited number of comparisons have been presented showing varying degrees of correlation between the experiment and associated finite element results. The pressure field produced by the finite element model over time did not predict the local cavitation phenomenon exactly, since the period of cavitation for the finite element model is less than that measured in the experiment. However, cavitation closure occurred within a few milliseconds of each other. Similarly, the displacement-time history of the centre of the plate in the finite element model shows an outward deflection of shorter duration than that observed in the experimental data, which again may be a result of premature cavitation closure in the finite element model.

Figures 8(a) and (b) show excellent agreement between the predicted displacement values of the finite element model with the experimental values. The accelerometer data exhibited far less scatter than the displacement transducer data and consequently, trends across the shock levels tested were far more apparent in this data. The primary reason for the scatter in opti- cal displacement transducer results is believed to have been the mounting arrangement used, which displayed looseness after each shock event. The electromechanical potentiometer type displacement transducer also suffered from an unsatisfactory mounting arrangement, which appeared to be a design fault that could not be readily corrected. However, the displacement transducers proved worthwhile for providing displacement-time histories over a far longer duration than the accelerometers, whose data contained significant drift following the test plate's initial shock response.

The accelerometer data were also integrated once, to produce peak velocity estimates for the test plate centre. The finite element model velocities tended to under predict the velocities calculated from the accelerometer data. One possibility is that the boundary conditions of the plate in the finite element model were fully clamped, whereas these conditions could not be fully met in the experiment. Since the plate motions were less restricted in the experiment, relatively higher peak velocities would have been achieved. However, discrepancies in the peak velocities are at odds with the excellent correlation between the experiment and finite element peak displacement values. This discrepancy is not readily explicable.

\section{Conclusions}

A quarter-symmetry LS-DYNA/USA finite element model was produced with the purpose of validating far-field underwater shock experiments.

Comparison of the experimental and finite element results show excellent agreement for peak displace- 
ments of the plate centre for the range of charge weight and standoff distances used. Less satisfactory correlation is observed for the peak velocity of the test plate centre, with significant deviation between experimental and finite element simulation results at higher shock levels.

The finite element model satisfactorily simulates the displacement-time history of the plate centre. The plate motions during the period of local cavitation, adjacent to the plate, are inaccurate, probably due to the finite element model's inability to accurately model the cavitation phenomenon.

It is expected that the ability of the finite element model to predict the experimental data will be significantly improved once hydrostatic initialisation can be fully utilised with the LS-DYNA/USA software.

The finite element model tends to under predict structural velocities. One reason may be that the finite element model assumes that the test plate is fully clamped whereas the experimental arrangement had less rigid boundary constraints (i.e. somewhere between simply supported and fully clamped). However, further work needs to be undertaken to confirm this argument.

\section{Acknowledgements}

Special thanks to Kendall Hook, Physics Dept, Royal Melbourne Institute of Technology, Australia for designing and constructing the Optical Displacement Transducer. Thanks also to the staff of the Specialised Instrumentation Group, Aeronautical and Maritime Research Laboratory, Defence Science and Technology Organisation, Australia who conducted the series of underwater experiments and provided high quality data.

\section{References}

[1] J.A. DeRuntz, The Underwater Shock Analysis Code and Its Applications, Proc. 60th Shock \& Vibration Symposium, 1989, pp. 89-107.

[2] J.A. DeRuntz, T.L. Geers and C.A. Felippa, The Underwater Shock Analysis Code (USA-Version 3), Defense Nuclear Agency, Washington, DC, Report 5615F, 1980.

[3] J.O. Hallquist, LS-DYNA User's Manual (Non-linear Dynamic Analysis of Solids in Three Dimensions), Livermore Software Technology Corporation, Report 1007, 1990. 

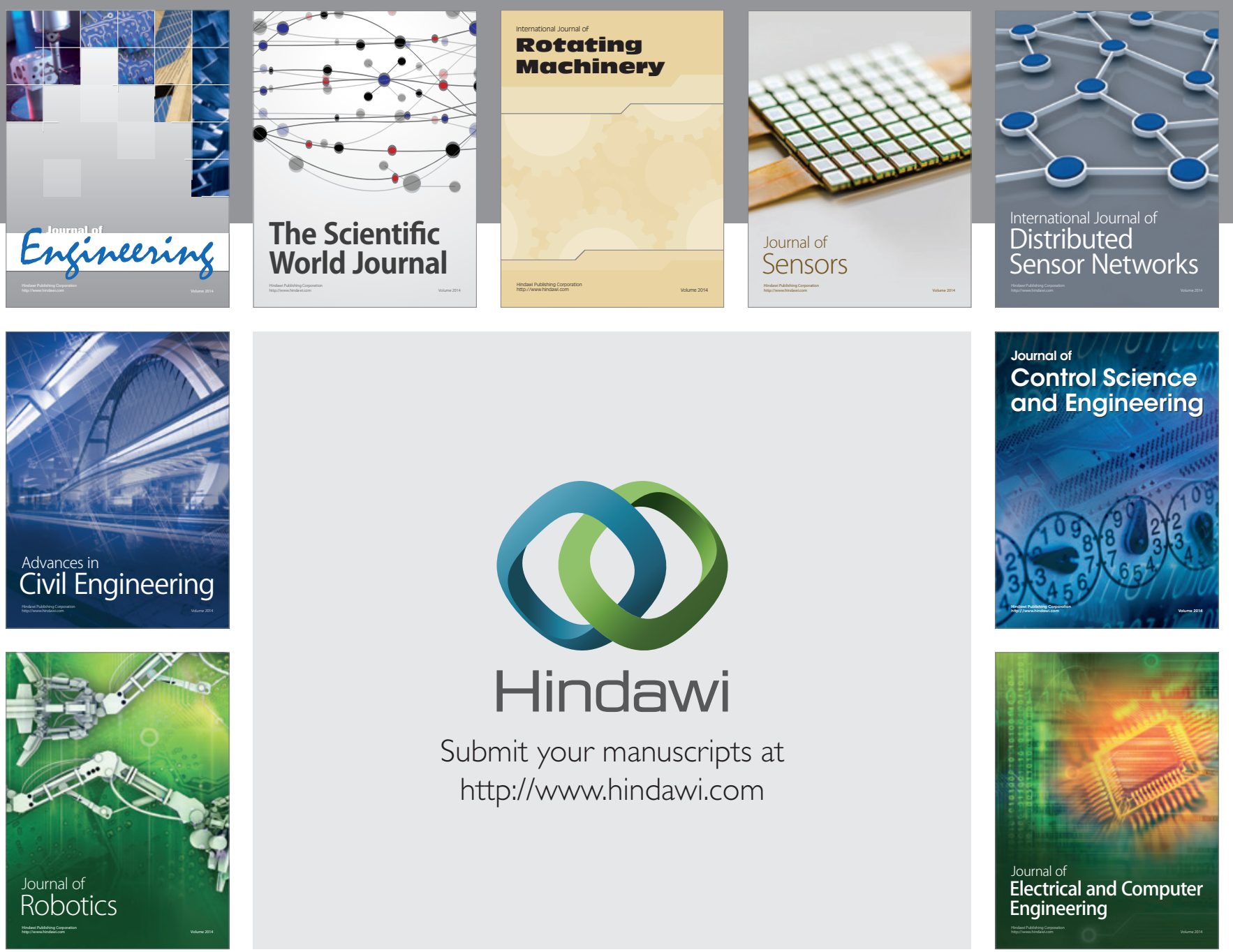

Submit your manuscripts at

http://www.hindawi.com
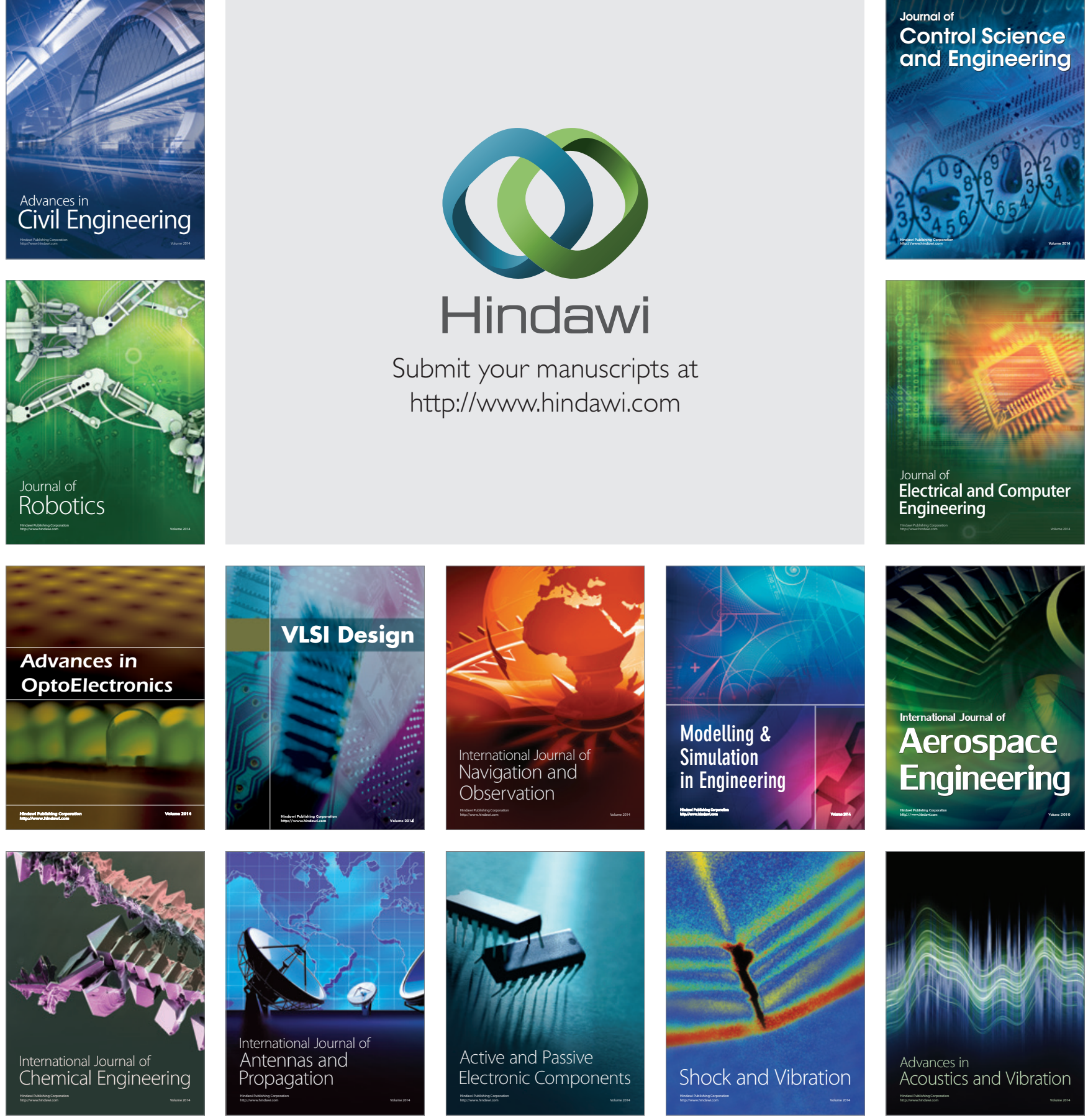